\title{
ESTIMATIVA DA CAPACIDADE DE CAMPO PELA CURVA DE RETENÇÃO E PELA DENSIDADE DE FLUXO DA ÁGUA ${ }^{(1)}$
}

\author{
Alexsandro dos Santos Brito ${ }^{(2)}$, Paulo Leonel Libardi ${ }^{(3)}$, Jaedson \\ Cláudio Anunciato Mota ${ }^{(4)}$ \& Sergio Oliveira Moraes ${ }^{(3)}$
}

\begin{abstract}
RESUMO
A capacidade de campo é um parâmetro de inegável relevância para o manejo adequado da irrigação. A partir da determinação confiável do conteúdo de água no solo na capacidade de campo, pode-se otimizar a produtividade das culturas agrícolas, maximizando a eficiência do uso da água pelas plantas e evitando a contaminação do lençol freático por lixiviação de fertilizantes e agroquímicos. Nesse sentido, o trabalho teve como objetivo avaliar a capacidade de campo (a) pelo cálculo da densidade de fluxo da água durante o processo de redistribuição no experimento clássico de determinação desse parâmetro e (b) a partir de uma dada tensão da água na curva de retenção, em um Latossolo Vermelho-Amarelo textura média, localizado em área experimental da Escola Superior de Agricultura Luiz de Queiroz, município de Piracicaba, Estado de São Paulo (Brasil). Para isso, instalaram-se no solo, até a profundidade de $1,9 \mathrm{~m}, 60$ tubos de acesso a uma sonda de nêutrons, numa rede de malha quadrada de $5 \mathrm{~m} \mathrm{(6} \times 10$ pontos amostrais), e, adjacente a cada tubo, dois tensiômetros, um a $0,75 \mathrm{~m}$ e outro a $0,85 \mathrm{~m}$ de profundidade. Nos 60 pontos (locais) amostrais, desenvolveu-se um experimento similar ao experimento clássico de determinação da capacidade de campo, no qual o solo foi devidamente submetido a uma lâmina de infiltração visando à saturação do seu perfil; sua superfície, coberta com lona plástica para evitar fluxo de água através dela; e a redistribuição da água, monitorada até a profundidade de $0,8 \mathrm{~m}$ durante 20 dias. Observou-se que o conteúdo de água correspondente a uma densidade de fluxo de $1,0 \mathrm{~mm} \mathrm{dia}{ }^{-1}$ é a melhor estimativa da capacidade de campo para esse solo, uma vez que as densidades de fluxo de 0,1 e $0,01 \mathrm{~mm}$ dia $^{-1}$, também recomendadas para estimar a capacidade de campo, são muito baixas, a ponto de não terem sido alcançadas neste estudo. Quanto aos resultados obtidos pelo método baseado na curva de retenção, utilizando-se o conteúdo de água correspondente à tensão de $10 \mathrm{kPa}$ em curvas de retenção elaboradas no campo e no laboratório
\end{abstract}

\footnotetext{
(1) Parte da Tese de Doutorado do primeiro autor (ESALQ/USP). Recebido para publicação em 23 de novembro de 2010 e aprovado em 17 de agosto de 2011.

(2) Professor efetivo do Instituto Federal de Educação, Ciência e Tecnologia Baiano, Campus Guanambi. E-mail: alexsandrobrt@gmail.com.

(3) Professor do Depto. de Engenharia de Biossistemas da ESALQ/USP. Av. Pádua Dias, 11, Piracicaba-SP, CEP13418-900. Email: pllibard@esalq.usp.br; somoraes@esalq.usp.br

(4) Bolsista DCR (CNPq/ FUNCAP) pela Universidade Federal do Ceará. E-mail: jaedsonmota@yahoo.com.br
} 
também para os 60 pontos, na profundidade de $0,8 \mathrm{~m}$, observou-se que os valores obtidos a partir das curvas de retenção elaboradas no campo e no laboratório subestimaram e superestimaram, respectivamente, aqueles baseados na densidade de fluxo da água de $1,0 \mathrm{~mm} \mathrm{dia}{ }^{-1}$, e a medida de campo foi mais confiável.

Termos de indexação: drenagem interna, potencial mátrico do solo, redistribuição de água.

\title{
SUMMARY: FIELD CAPACITY ESTIMATION BASED ON RETENTION CURVE AND SOIL WATER FLUX DENSITY
}

\begin{abstract}
Field capacity is a parameter of undeniable importance for an adequate irrigation management. From the reliable determination of soil water content and field capacity, crop yields cam be optimized, maximizing the plant water use efficiency and preventing groundwater contamination by leaching of fertilizers and agrochemicals. The objective of this study was to evaluate field capacity (a) from the calculation of soil water flux density during water redistribution in a classical experiment to determine this parameter and (b) from a given soil water tension in the retention curve. The soil was a medium texture Red Yellow Latosol, in an experimental area of the Escola Superior de Agricultura "Luiz de Queiroz", county of Piracicaba,

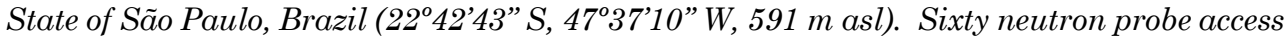
tubes were installed in the soil profile in a $5 \mathrm{~m}$ square mesh grid ( $6 x 10$ sampling points) and adjacent to each tube two tensiometers (depth of 0.75 and $0.85 \mathrm{~m}$ ). At the 60 sampling points, a similar experiment as described above was carried out, in which a great amount of water was applied to the soil surface to saturate the profile to a depth of $0.8 \mathrm{~m}$. Then the soil surface was covered with a plastic tarpaulin to avoid water flows. Water redistribution was monitored in the 0.0-0.8 m layer for 20 days. The best estimation of field capacity for this soil was the soil water content at a soil water flux density of $1.0 \mathrm{~mm}_{\text {day-1 }}$, since the flux densities of 0.1 and $0.01 \mathrm{~mm}^{\mathrm{m}} \mathrm{day}^{-1}$, also recommended to estimate field capacity, are so low that they were not even reached in this study. By the method based on the soil water retention curve, using the soil water content at a tension of $10 \mathrm{kPa}$ in soil water retention curves determined in field and laboratory, also for the 60 experimental points (depth of $0.8 \mathrm{~m}$ ), it was observed that the values obtained from field retention curves were lower and values obtained from laboratory retention curves were higher than those based on a flux density of $1.0 \mathrm{~mm}^{\text {day-1 }}{ }^{-1}$ in comparison, the field measurements were more consistent.
\end{abstract}

Index terms: Internal drainage, soil matric potential, water redistribution.

\section{INTRODUÇÃO}

O termo capacidade de campo foi criado por Veihmeyer \& Hendricson (1931), numa tentativa de oferecer maior aplicabilidade ao conceito "equivalente de umidade" introduzido por Briggs \& McLane (1910), como descrevem Twarakavi et al. (2009). O equivalente de umidade foi definido como a percentagem de água que um solo pode reter contra uma "força centrífuga" de 1.000 vezes a "força da gravidade" por $30 \mathrm{~min}$. Por outro lado, a capacidade de campo representa o conteúdo de água retida no solo após o excesso de água ter sido drenado e o movimento da água tornar-se desprezível (Meyer \& Gee, 1999). Pela equação de Darcy-Buckingham, a condutividade hidráulica é o principal fator de redução da densidade de fluxo da água no solo e torna-se nula apenas quando o conteúdo de água no solo for zero - situação praticamente impossível (Reichardt, 1988).
Durante os processos de infiltração e drenagem, as principais forças que alteram o movimento da água são a da gravidade e a da capilaridade. O excesso de água refere-se ao volume de água que ocupa a macroporosidade e que é redistribuído no perfil do solo pela força da gravidade, podendo atingir grande magnitude. A partir desse momento, a capilaridade passa a atuar predominantemente e, dependendo das características físico-hídricas do solo, o movimento de água para baixo ainda pode ter um valor expressivo. A diminuição desse movimento é mais rápida para um solo com textura arenosa, em comparação ao de textura média ou argilosa (Nachabe, 1998).

Pela própria definição, observa-se que a capacidade de campo é um estado hídrico específico para cada tipo de solo e observado durante o processo de redistribuição da água. Para a escala do perfil de solo explorado pelas culturas agrícolas, existe uma dependência muito forte entre as características 
físicas, físico-hídricas e químicas, ligadas principalmente às distribuições de tamanho de partículas e de poros e à quantidade de matéria orgânica dos horizontes pedológicos. Numa escala mais ampla, as profundidades do solo e do lençol freático são fatores influentes na determinação da capacidade de campo (Meyer \& Gee, 1999).

O momento em que o movimento da água para baixo torna-se desprezível é também caracterizado por certo potencial mátrico e tempo de redistribuição. Em virtude dessas correlações e da facilidade que esses parâmetros trazem para a determinação da capacidade de campo, os pesquisadores, em todo o mundo, têm desenvolvido trabalhos com o objetivo de aprimorar os métodos baseados no tempo e no potencial mátrico. Para o método baseado na densidade de fluxo da água, os valores de $1,0,1$ e $0,01 \mathrm{~mm}^{2} \mathrm{di}^{-1}$ são os mais recomendados para a caracterização da capacidade de campo (Meyer \& Gee, 1999; Twarakavi et al., 2009). Quanto ao método baseado no tempo de redistribuição, tem-se que para os solos com textura arenosa atingese a capacidade de campo em torno de três dias, enquanto para solos com textura média e argilosa ela é alcançada entre seis a oito dias, quando o perfil do solo é previamente submetido a uma lâmina de infiltração, na tentativa de atingir a saturação (Twarakavi et al., 2009).

Richards \& Weaver (1944) observaram que, em 71 solos, o equivalente de umidade correlacionou-se com o conteúdo de água equilibrado no potencial mátrico de $-33 \mathrm{kPa}$. A partir de trabalhos realizados para solos de regiões temperadas, com características físico-hídricas e morfológicas diferentes daquelas das regiões tropicais, passou-se a adotar o conteúdo de água correspondente ao potencial mátrico de $-33 \mathrm{kPa}$ como representativo da capacidade de campo, devido à facilidade de obtenção em laboratório, independentemente das características físico-hídricas e morfológicas dos solos. Em regiões temperadas, o conteúdo de água no potencial mátrico de $-33 \mathrm{kPa}$ correlaciona-se bem com a capacidade de campo, devido à presença de argila com atividade alta (Ruiz et al., 2003). Alguns solos com textura argilosa também apresentam boa correlação com esse potencial mátrico (Jabro et al., 2009). Entretanto, para solos argilosos que apresentam horizonte $\mathrm{B}$ textural, a adoção desse potencial mátrico subestima a capacidade de campo (Reichardt, 1988), devido ao impedimento do fluxo e consequente estagnação da água na camada superficial do solo. Para solos arenosos e aqueles localizados nas regiões tropicais, os potenciais mátricos de - 6 e - $10 \mathrm{kPa}$ são mais realistas (Reichardt, 1988; Meyer \& Gee, 1999). Como se trata de um conteúdo de água atingido durante os primeiros dias de um processo dinâmico, a influência da estrutura do solo é muito grande, ao contrário do que ocorre para o ponto de murcha permanente, no qual a influência da distribuição do tamanho das partículas passa a ser preponderante.

Atualmente, funções de pedotransferência têm sido utilizadas para estimar a capacidade de campo (Givi et al., 2004), por meio dos parâmetros de distribuição do tamanho das partículas, densidade do solo, conteúdo de matéria orgânica e parâmetros de retenção da água no solo. Entretanto, a precisão da maioria dessas funções não é boa, o que dificulta a aplicabilidade para manejo correto das culturas agrícolas.

A determinação adequada da capacidade de campo é fundamental para o correto manejo das culturas agrícolas, principalmente em áreas irrigadas, e para a otimização de manejos de solo e da irrigação, visando maximizar a eficiência de uso da água pelas plantas. Atrelado a esse aspecto, tem-se ainda que a utilização de um valor alto de capacidade de campo, estimado para outros solos, pode provocar drenagem interna excessiva e causar lixiviação de fertilizantes e agroquímicos. Portanto, o objetivo deste trabalho foi avaliar a capacidade de campo pelo cálculo da densidade de fluxo da água, desenvolvendo um experimento similar ao experimento clássico para sua determinação no campo, e pelo conteúdo de água correspondente a uma dada tensão da água na curva de retenção, determinada no campo e no laboratório, em um Latossolo Vermelho-Amarelo textura média.

\section{MATERIAL E MÉTODOS}

O estudo foi realizado em uma área experimental da Escola Superior de Agricultura Luiz de Queiroz, localizada no município de Piracicaba, Estado de São Paulo, Brasil. As coordenadas geográficas da área são $22^{\circ} 42^{\prime} 43^{\prime}$ S, $47^{\circ} 37^{\prime} 10^{\prime \prime} \mathrm{W}$ e $591 \mathrm{~m}$ de altitude. O solo foi classificado como Latossolo Vermelho-Amarelo distrófico (Embrapa, 2006), relevo plano ondulado (Figura 1), no qual foram instalados 60 tubos de acesso a uma sonda de nêutrons (modelo 503 Hydroprobe da $\mathrm{CPN}^{\odot}$, com fonte de Amerício-Berílio $50 \mathrm{~m} \mathrm{Ci}$ ), numa rede de malha quadrada com pontos distanciados de $5 \mathrm{~m}\left(6 \times 10\right.$ pontos, totalizando $\left.1.500 \mathrm{~m}^{2}\right)$. Os tubos de acesso eram de alumínio e tinham comprimento de 2,10 m (0,2 m acima da superfície), diâmetro interno de 0,045 m e espessura de parede de 0,0015 m.

A calibração da sonda de nêutrons foi feita na área experimental. Para isso, foram realizadas 13 varreduras, em intervalos de 14 dias, procedendo-se às contagens (leituras da sonda) em todos os locais amostrais e nas profundidades de 0,$2 ; 0,4 ; 0,6$; e $0,8 \mathrm{~m}$. Para buscar medidas que cobrissem a maior faixa de conteúdo de água possível, foram selecionadas oito dessas 13 varreduras. Nessas oito varreduras foram feitas três repetições de leituras com a sonda de nêutrons nas profundidades e na posição-padrão, ao passo que a coleta de amostras de solo com estrutura deformada, para medida do conteúdo de água no solo, foi feita sempre a uma distância de $0,4 \mathrm{~m}$ do tubo de acesso à sonda de nêutrons.

O conteúdo de água à base de massa, $U$, nos locais e profundidades selecionados, determinado após 


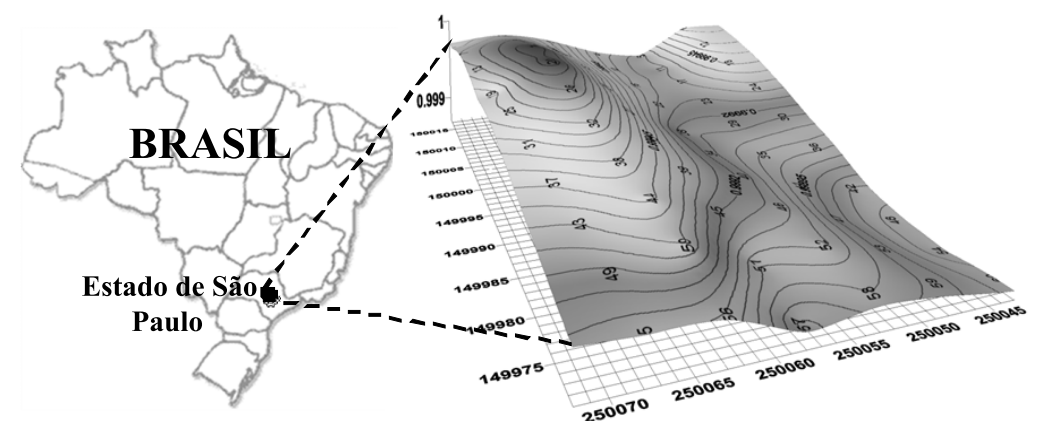

Figura 1. Localização e mapa planialtimétrico da área experimental (UTM), construído com a cota relativa à maior cota e apresentando a distribuição dos locais amostrais.

secagem em estufa a $105^{\circ} \mathrm{C}$, durante $24 \mathrm{~h}$, foi transformado em conteúdo de água à base de volume, $\theta$, utilizando-se a densidade do solo, $\rho$, determinada nas mesmas profundidades e para cada um dos pontos amostrais, conforme equação:

$$
\theta=\mathrm{U} \times\left(\rho / \rho_{\mathrm{a}}\right)
$$

em que $\rho_{a}$ é a densidade da água, considerada constante e igual a $1.000 \mathrm{~kg} \mathrm{~m}^{-3}$.

Dessa forma, elaboraram-se as equações de calibração da sonda de nêutrons para as referidas profundidades, por meio de regressão linear simples da contagem relativa (razão entre a contagem na posição de amostragem e a contagem na posiçãopadrão), em função do conteúdo de água no solo (Figura 2).

A determinação da densidade do solo foi realizada pelo método do anel volumétrico, utilizando um extrator tipo Uhland, com anéis volumétricos medindo $0,05 \mathrm{~m}$ de altura e de diâmetro. Foram coletadas amostras em todos os locais amostrais e nas referidas profundidades, totalizando 240 amostras. A distribuição do tamanho das partículas também foi determinada em todos os pontos amostrais e profundidades, pelo método da pipeta (Gee \& Or, 2002).

O estudo sobre retenção da água no solo foi realizado a partir de curvas de retenção na profundidade de 0,8 m, utilizando métodos de laboratório e de campo. Para o método de laboratório, utilizaram-se funis de Haines para as tensões de 1 a $10 \mathrm{kPa}$, com medições a cada $1 \mathrm{kPa}$, totalizando dez pontos, e câmaras de Richards para as tensões de 30, 50, 70 e $100 \mathrm{kPa}$. Para as curvas elaboradas em campo, utilizaram-se medidas de potencial mátrico com tensiômetros e conteúdos de água estimados com a sonda de nêutrons ao longo do desenvolvimento do trabalho. Para isso, utilizaram-se dois tensiômetros instalados nas profundidades de 0,75 e $0,85 \mathrm{~m}$, sendo calculada a média entre as medidas, como representativa do potencial mátrico na profundidade de $0,8 \mathrm{~m}$. Observase que a média das medidas de tensão a 0,75 e 0,85 m aproxima o tamanho da amostra para essas medidas daquele para as estimativas do conteúdo de água. Para o ajuste das curvas de retenção, utilizou-se o modelo proposto por van Genuchten (1980) e o programa computacional Table Curve 2D (Jandel Scientific, Copyright 1989-1994), versão 5.01 (Trial).
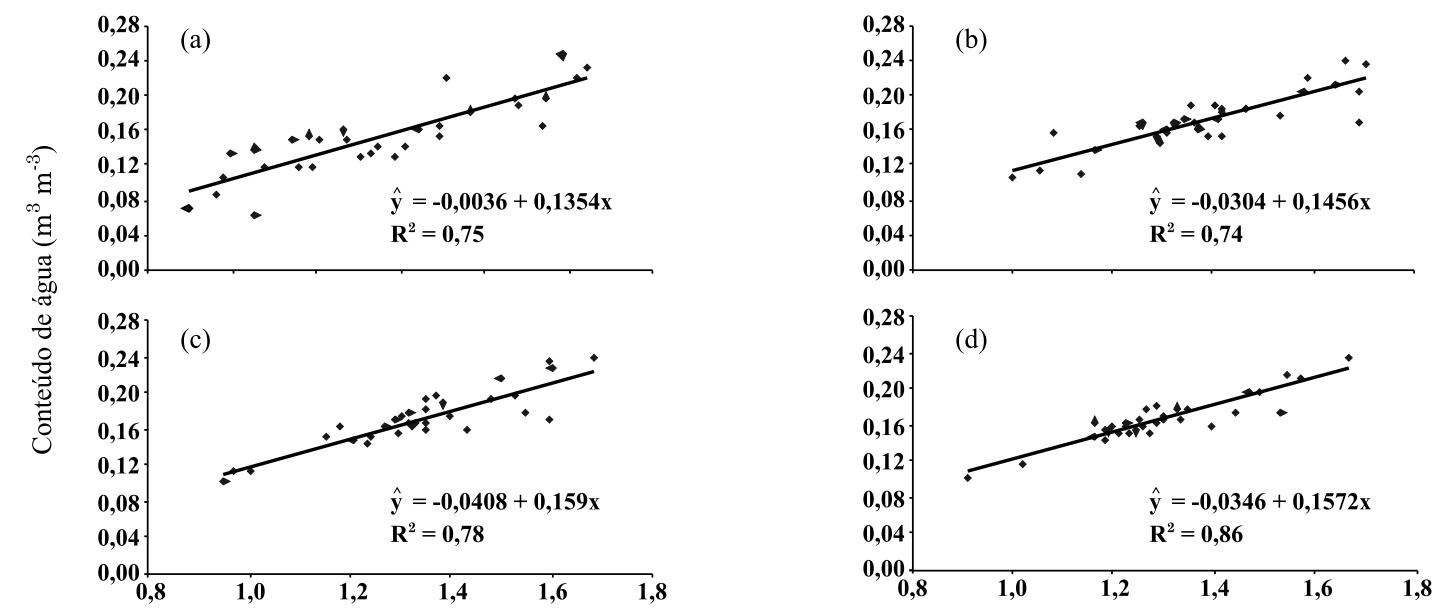

Contagem relativa média

Figura 2. Curvas de calibração da sonda de nêutrons para as profundidades de 0,20 (a), 0,40 (b), 0,60 (c) e $0,80 \mathrm{~m}(\mathrm{~d})$. 
Com as curvas de retenção, feitas no laboratório e no campo, determinou-se a capacidade de campo como sendo o conteúdo de água correspondente ao potencial mátrico de $-10 \mathrm{kPa}$.

A determinação da capacidade de campo pelo método baseado na densidade de fluxo da água foi também realizada nos 60 locais amostrais (Figura 1), nos quais se instalou uma chapa galvanizada $(2 \mathrm{~mm}$ de espessura) até a profundidade de $0,40 \mathrm{~m}$ (deixando $0,20 \mathrm{~m}$ da altura da chapa acima da superfície do solo, para manutenção de uma lâmina de água de mesma altura, propiciando a infiltração da água no solo) e com diâmetro de $3,0 \mathrm{~m}$, com o objetivo de se obter apenas fluxo vertical, sobretudo na porção central dessa coluna de solo no campo.

Cada coluna de solo recebeu água até alcançar o maior conteúdo desta, indicado pela maior contagem detectada na sonda de nêutrons e a menor leitura dos tensiômetros a 0,75 e $0,85 \mathrm{~m}$ de profundidade. A lâmina de infiltração foi mantida com nível constante até que se registrasse contagem constante e os tensiômetros mostrassem leitura que indicasse a saturação. Após os indicativos de saturação, cessavase o fornecimento de água e, antes de infiltrar toda a lâmina, procedia-se à leitura com a sonda de nêutrons e dos manômetros de mercúrio dos tensiômetros, para que se conseguisse medir o conteúdo de água de saturação em campo, uma vez que o processo de drenagem é muito rápido no solo estudado. Após a lâmina de água ter se infiltrado, colocou-se uma lona impermeável sobre o solo, com as bordas enterradas nos limites da coluna de solo, e outra lona (lonil) com a finalidade de evitar a entrada (precipitação pluvial) e a saída (evaporação) de água, visto que se desejava estudar o processo de redistribuição da água no solo.

As leituras da sonda de nêutrons para obtenção do conteúdo de água envolveram três repetições em cada profundidade e em função do tempo de redistribuição da água no solo, sempre realizando a leitura da contagem na posição-padrão. Cada parcela experimental foi monitorada por 20 dias, uma vez que para esse solo a redistribuição da água no perfil, partindo de um conteúdo de água próximo da saturação, praticamente cessa com 18 a 20 dias.

Quanto ao cálculo da densidade de fluxo da água em cada parcela experimental, utilizou-se a equação da continuidade integrada com relação à profundidade de solo. Essa equação, pela configuração do experimento em que a densidade de fluxo da água é nula na superfície do solo durante o processo de redistribuição, torna-se:

$$
q_{z}=-\int_{0}^{Z} \frac{\partial \theta}{\partial t} d Z
$$

em que $\theta$ é o conteúdo de água, $t$ é o tempo de redistribuição da água e $\mathrm{q}_{\mathrm{z}}$ é a densidade de fluxo da água na profundidade de solo $Z$.
A capacidade de campo foi avaliada pelas densidades de fluxo de 0,$01 ; 0,1 ;$ e $1,0 \mathrm{~mm} \mathrm{dia}^{-1}$ nas profundidades de 0,$2 ; 0,4 ; 0,6$; e $0,8 \mathrm{~m}$; para a profundidade de $0,8 \mathrm{~m}$, procedeu-se a comparações dos valores do conteúdo de água correspondentes à tensão da água de $10 \mathrm{kPa}$, determinados a partir das curvas de retenção feitas no laboratório e no campo. A armazenagem de água ( $0-0,8 \mathrm{~m})$ ao longo do tempo de sua redistribuição no solo e a capacidade de campo relativa $-C C_{r}$ (Reynolds et al., 2008), nas quatro profundidades estudadas, também foram avaliadas:

$$
C C_{r}=\frac{C C}{\theta_{s}}
$$

em que $C C$ é o conteúdo de água no solo à capacidade de campo e $\theta_{\mathrm{s}}$ é o conteúdo de água de saturação, assumindo as CC correspondentes às densidades de fluxo da água de 0,$01 ; 0,1$; e 1,0 $\mathrm{mm} \mathrm{dia}^{-1}$.

\section{RESULTADOS E DISCUSSÃO}

O relevo do terreno é considerado plano, porém com a presença de porções mais altas (Figura 1). Com o mapa planialtimétrico representado por cotas altimétricas relativas à maior cota, foi possível a observação de pequenas depressões, com diferença de $0,76 \mathrm{~m}$ entre a maior e a menor cota.

Analisando o mapa planialtimétrico em conjunto com a distribuição do tamanho das partículas (Figura 3), é possível observar maior quantidade de areia nas profundidades de 0,2 e $0,4 \mathrm{~m}$ nos locais amostrais posicionados nas maiores cotas do terreno, enquanto aqueles posicionados em cotas menores apresentam maior quantidade de argila. Para as profundidades de 0,6 e 0,8 m, observa-se grande uniformidade das frações granulométricas, decorrente da homogeneidade na ação dos fatores e processos de formação do solo.

Quanto à densidade do solo, como a área estava em pousio, sem cultivos e com vegetação espontânea há quatro anos - e como não foi feita nenhuma prática de preparo, apenas algumas passadas com roçadeira para limpeza -, a camada superficial $(0-0,3 \mathrm{~m})$ apresentou densidade mais elevada, evidenciando um processo de acomodação (Quadro 1), uma vez que em solos arenosos, como é o caso da camada superficial desse solo, a densidade pode variar de 1.250 a $1.750 \mathrm{~kg} \mathrm{~m}^{-3}$ (Brady \& Weil, 2002).

A porosidade do solo na profundidade de $0,8 \mathrm{~m}$ apresenta-se em bom estado, com predominância de microporos, mas com $23,9 \%$ de macroporos, o que torna esse solo muito drenável, principalmente quando próximo à saturação.

Os parâmetros de ajuste (Quadro 2) do modelo de van Genuchten (1980) para as curvas de retenção de água elaboradas com dados de campo e de laboratório 

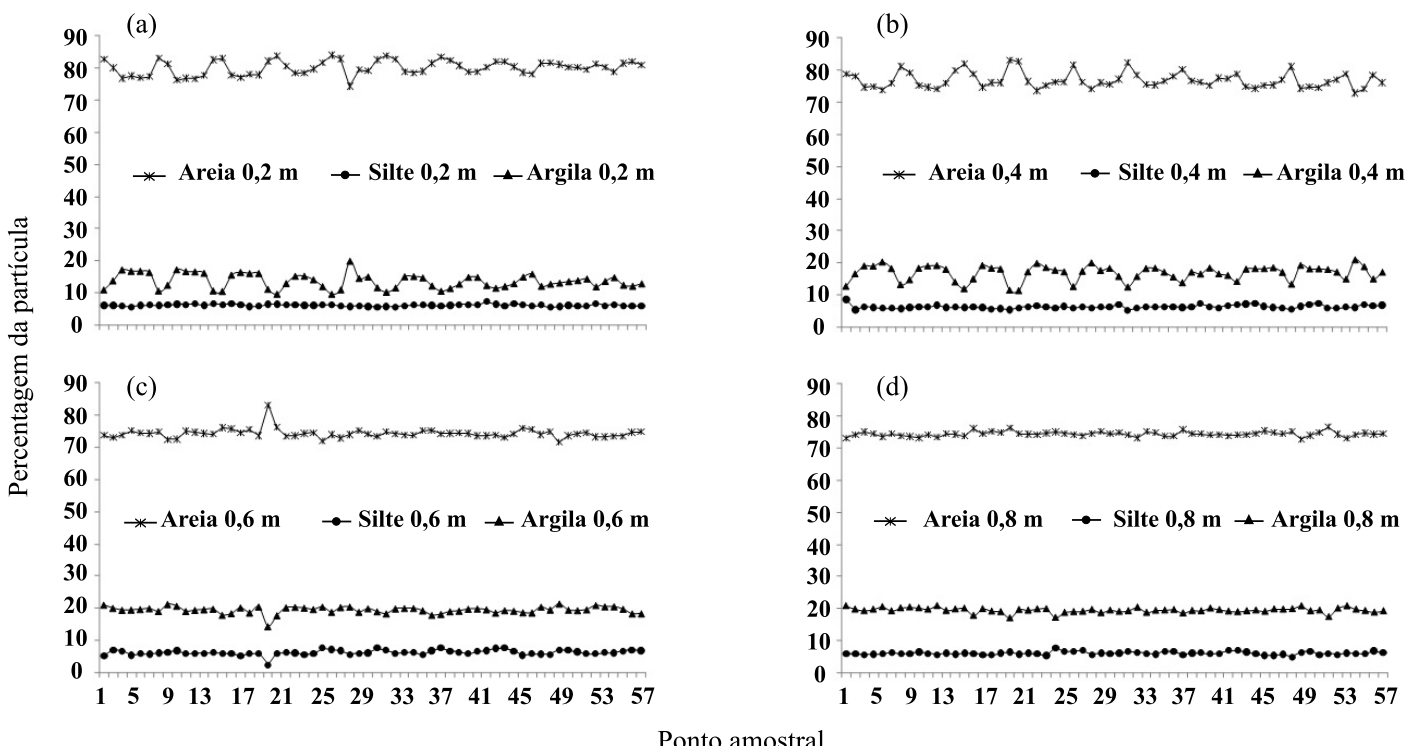

Figura 3. Percentagem de areia, silte e argila nas profundidades de 0,2 m (a), 0,4 m (b), 0,6 m (c) e 0,8 m (d), nos 60 locais amostrais.

Quadro 1. Densidade do solo em função de sua profundidade (m) e porosidade (\%), na profundidade de $0,8 \mathrm{~m}$

\begin{tabular}{|c|c|c|c|c|c|c|c|c|}
\hline & \multicolumn{4}{|c|}{ Densidade do solo $\left(\mathrm{kg} \mathrm{m}^{-3}\right)$} & \multicolumn{4}{|c|}{ Porosidade do solo $(\%)^{(3)}$} \\
\hline & $0,2 \mathrm{~m}$ & $0,4 \mathrm{~m}$ & $0,6 \mathrm{~m}$ & $0,8 \mathrm{~m}$ & Macro & Meso & Micro & Total \\
\hline Média ${ }^{(1)}$ & 1674 & 1610 & 1510 & 1509 & 10,45 & 12,56 & 20,76 & 43,77 \\
\hline $\mathrm{EPM}^{(2)}$ & 6,00 & 9,67 & 6,49 & 3,94 & 0,17 & 0,32 & 0,17 & 0,19 \\
\hline Máxima & 1771 & 1799 & 1619 & 1598 & 15,25 & 17,41 & 25,83 & 47,81 \\
\hline Mínima & 1568 & 1400 & 1372 & 1444 & 6,84 & 7,20 & 18,38 & 39,71 \\
\hline
\end{tabular}

(1) Médias originadas dos 60 locais amostrais. ${ }^{(2)} \mathrm{EPM}=$ erro-padrão da média. ${ }^{(3)}$ Porosidade total $(\alpha)$ calculada por $\quad \alpha=1-\left(\rho / \rho_{\mathrm{s}}\right)$, sendo $\rho_{\mathrm{s}}$ a densidade das partículas e igual a $2.684 \mathrm{~kg} \mathrm{~m}^{-3}$ e macro, meso e microporos classificados segundo Kooveraar et al. (1983).

são bastante diferentes, pelo fato, principalmente, de as condições de contorno serem diferentes nos dois métodos de construção da curva de retenção da água no solo. Nos dados de campo, apesar de os potenciais mátricos mais altos terem sido obtidos quando o solo se encontrava numa condição propícia à saturação (perfil do solo sob uma lâmina de infiltração de $0,20 \mathrm{~m}$ e processo de inundação no qual foram aplicados aproximadamente $4.000 \mathrm{~L}$ de água), é muito provável que não se consiga uma saturação, como acontece no laboratório com uma pequena amostra com estrutura indeformada. Entretanto, pode-se considerar que esse conteúdo de água é o máximo que pode ocorrer no solo em condições naturais, sendo, portanto, uma condição muito mais próxima daquela que pode acontecer em eventos chuvosos intensos e prolongados.

Os perfis de conteúdo de água inicial e final dos 60 locais amostrais construídos para o período de redistribuição da água no solo (Figura 4) possuem baixa variação, em decorrência da uniformidade do solo. A presença de pontos mais baixos no terreno (Figura 1) e com maior quantidade de argila (Figura 3) não ocasionou aumento da variabilidade dos conteúdos de água inicial e final; o coeficiente de variação médio dos conteúdos de água, em todas as profundidades, foi de aproximadamente $7,3 \%$. Melo Filho \& Libardi (2005) também verificaram esse comportamento para um Latossolo semelhante ao estudado. Outro destaque é o perfil do conteúdo de água inicial relativamente baixo, com o valor médio de $0,25 \mathrm{~m}^{3} \mathrm{~m}^{-3}$, pelo fato de possuir um conteúdo de argila médio de 137, 168, 194 e $195 \mathrm{~g} \mathrm{~kg}^{-1}$ nas profundidades de 0,$2 ; 0,4 ; 0,6$; e $0,8 \mathrm{~m}$, respectivamente.

A condição do perfil de solo previamente saturado é fundamental para a determinação confiável da capacidade de campo. Embora a definição de capacidade de campo com base no tempo de redistribuição da água seja usada largamente, a sua validade precisa ser investigada apropriadamente (Twarakavi et al., 2009). 
Quadro 2. Parâmetros de ajuste das curvas de retenção para a profundidade de 0,8 m, segundo o modelo van Genuchten (1980)

\begin{tabular}{|c|c|c|c|c|c|c|c|c|c|c|}
\hline & \multicolumn{5}{|c|}{ Parâmetros da curva de retenção no campo } & \multicolumn{5}{|c|}{ Parâmetros da curva de retenção no laboratório } \\
\hline & $\alpha$ & $\mathrm{n}$ & $\mathrm{m}$ & $\theta_{\mathrm{r}}$ & $\theta_{\mathrm{s}}$ & $\alpha$ & $\mathrm{n}$ & $\mathrm{m}$ & $\theta_{\mathrm{r}}$ & $\theta_{\mathrm{s}}^{(3)}$ \\
\hline Média ${ }^{(1)}$ & 1,858 & 0,219 & 11,417 & 0,149 & 0,269 & 0,266 & 3,614 & 0,341 & 0,124 & 0,359 \\
\hline $\mathrm{EPM}^{(2)}$ & 0,043 & 0,003 & 0,162 & 0,002 & 0,003 & 0,006 & 0,109 & 0,030 & 0,002 & 0,003 \\
\hline Máxima & 2,540 & 0,264 & 13,751 & 0,185 & 0,366 & 0,336 & 6,122 & 1,837 & 0,147 & 0,412 \\
\hline Mínima & 0,857 & 0,153 & 7,995 & 0,054 & 0,237 & 0,066 & 1,496 & 0,084 & 0,075 & 0,314 \\
\hline
\end{tabular}

(1) Médias originadas dos 60 locais amostrais. ${ }^{(2)} \mathrm{EPM}=$ erro-padrão da média. ${ }^{(3)} \theta_{\mathrm{s}}$ introduzido como parâmetro de ajuste.

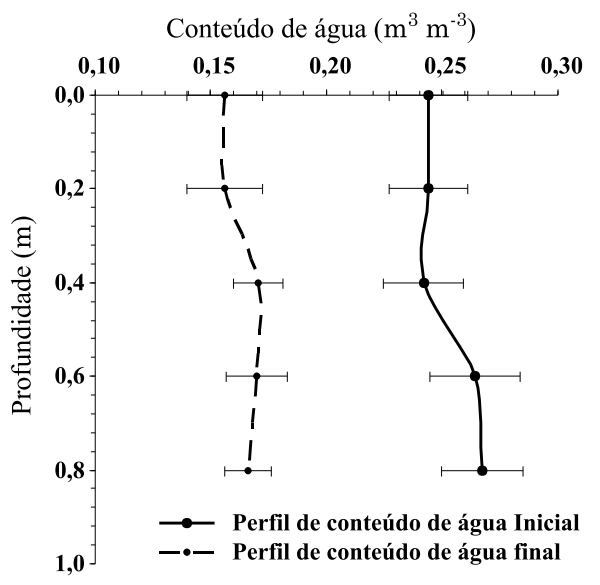

Figura 4. Perfis de conteúdo de água inicial e final dos processos de redistribuição da água no solo para os 60 locais amostrais e os respectivos desvios-padrão. O conteúdo de água na superfície do solo foi considerado igual ao da profundidade de $0,2 \mathrm{~m}$.
Para o tempo de redistribuição da água no solo de aproximadamente 480 horas, o conteúdo de água na capacidade de campo, correspondente a uma densidade de fluxo da água de $1,0 \mathrm{~mm} \mathrm{dia}^{-1}$, foi alcançado em 52, 97, 152 e 205 horas, para as profundidades de 0,2; 0,4; 0,6; e 0,8 m, respectivamente (Figura 5).

Considerando a profundidade do solo de $0,2 \mathrm{~m}$, o tempo necessário para atingir a capacidade de campo foi menor do que as estimativas reportadas na literatura (Twarakavi et al., 2009). O tempo necessário para atingir a capacidade de campo em solos com textura contrastante, com base no tempo decorrido após o processo de infiltração e no gradiente de potencial mátrico aproximadamente zero, foi de 50 e $450 \mathrm{~h}$ para solos franco-arenoso e franco-argiloso, respectivamente (Jabro et al., 2009). O solo franco-arenoso é semelhante ao avaliado neste trabalho em termos de distribuição do tamanho das partículas e da densidade do solo, demonstrando a similaridade entre os resultados quando da utilização de métodos de campo baseados no processo de redistribuição da água no solo.

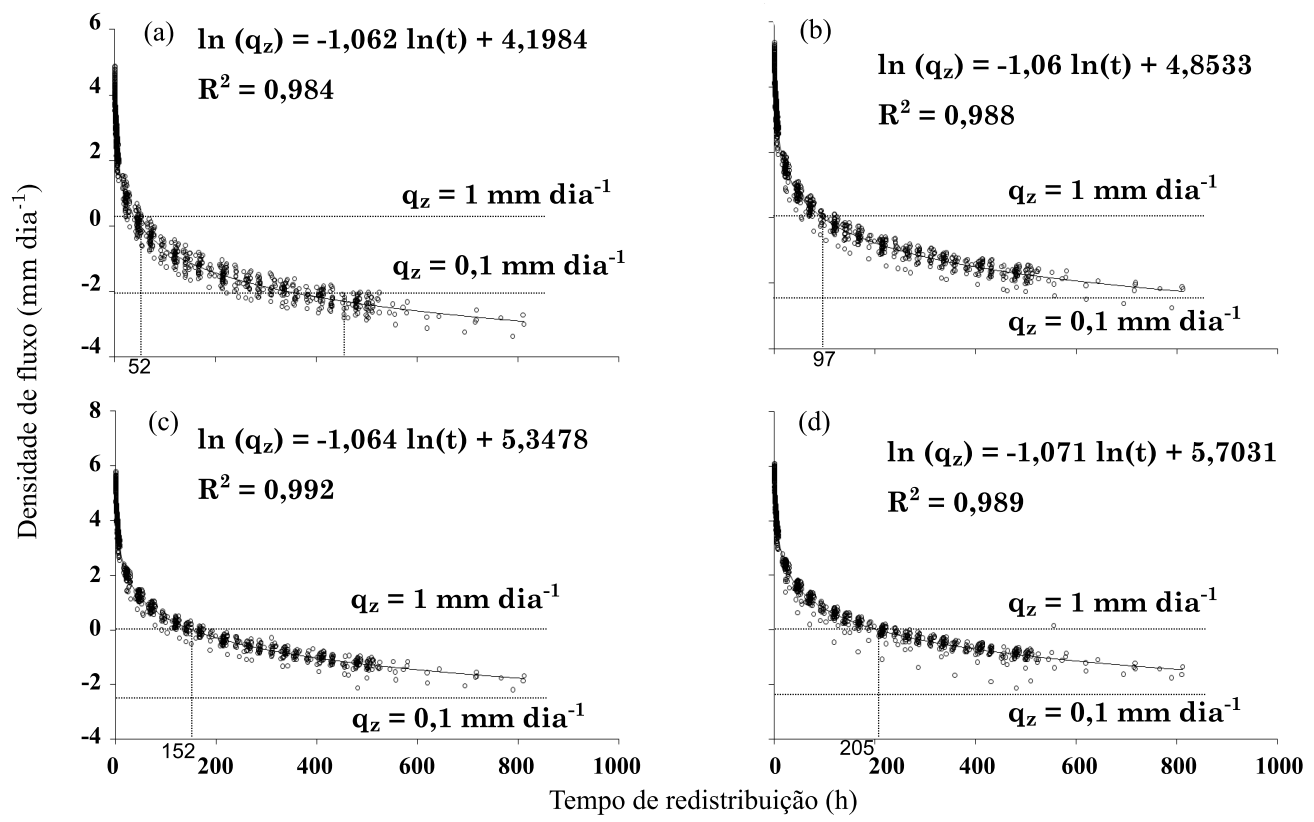

Figura 5. Densidade de fluxo da água $\left(\operatorname{Ln} q_{z}\right)$ em função do tempo de redistribuição da água no solo, determinada pela equação (2) nas profundidades (m) de 0,2 (a), 0,4 (b), 0,6 (c) e 0,8 (d), em 60 repetições e contendo 965 pontos. 
A densidade de fluxo da água de $0,1 \mathrm{~mm} \mathrm{dia}^{-1}$ foi atingida apenas na camada superficial do solo (profundidade de 0,2 m) e, mesmo assim, após $455 \mathrm{~h}$ de redistribuição (Figura 5a), o que se deve, possivelmente, à acomodação natural do solo, em virtude de ele não ter sido cultivado por alguns anos (Quadro 1), uma vez que a alta quantidade de areia na camada superficial seria um fator propício à alta permeabilidade. Observa-se que a inclinação da porção reta da curva de densidade de fluxo em função do tempo de redistribuição torna-se menor com o aumento da profundidade do solo. Dessa forma, as densidades de fluxo de 0,1 e $0,01 \mathrm{~mm} \mathrm{dia}^{-1}$ mencionadas na literatura (Meyer \& Gee, 1999; Twarakavi et al., 2009) não são recomendadas para estimar a capacidade de campo para esse Latossolo Vermelho-Amarelo, para o qual a melhor estimativa foi alcançada em uma densidade de fluxo de 1,0 $\mathrm{mm} \mathrm{dia}^{-1}$.

A partir de equações de regressão da densidade de fluxo em função do conteúdo de água no solo, as capacidades de campo correspondentes às densidades de fluxo de 1,0, 0,1 e 0,01 mm dia ${ }^{-1}$ foram, em média $(\mathrm{n}=60)$, de: $0,179,0,152$ e $0,125 \mathrm{~m}^{3} \mathrm{~m}^{-3}$ na profundidade de $0,2 \mathrm{~m} ; 0,185,0,161$ e $0,136 \mathrm{~m}^{3} \mathrm{~m}^{-3}$ na profundidade de $0,4 \mathrm{~m} ; 0,181,0,15$ e $0,119 \mathrm{~m}^{3} \mathrm{~m}^{-3}$ na profundidade de 0,60 m; e 0,173, 0,139 e $0,106 \mathrm{~m}^{3} \mathrm{~m}^{-3}$ na profundidade de $0,8 \mathrm{~m}$ (Figura 6a). Quando se compara a capacidade de campo baseada no potencial mátrico de $-10 \mathrm{kPa}$ com a obtida, assumindo-se a densidade de fluxo de $1,0 \mathrm{~mm} \mathrm{dia}^{-1}$, verifica-se que, quando a curva de retenção foi obtida em laboratório, com amostras indeformadas, houve superestimativa em relação à curva obtida no campo (Figura 6b).

As divergências quanto ao método de confecção da curva de retenção podem ser devido à combinação de ar aprisionado no solo e, ou, alterações na densidade do solo, nas amostras utilizadas no laboratório, principalmente em solos com baixa capacidade de retenção de água (Morgan et al., 2001), como é o caso do solo estudado neste trabalho.

Analisando a densidade de fluxo da água em função do potencial mátrico na profundidade de $0,8 \mathrm{~m}$ ao longo do tempo de redistribuição da água no solo (Figura 7), observa-se que o valor de $1,0 \mathrm{~mm} \mathrm{dia}^{-1}\left(\mathrm{Ln} \mathrm{q}_{\mathrm{z}}=0\right.$ é igual $\left.\mathrm{a}_{\mathrm{z}}=1,0 \mathrm{~mm} \mathrm{dia}^{-1}\right)$ corresponde, aproximadamente, ao potencial mátrico de $-10 \mathrm{kPa}(=-1,0 \mathrm{mca})$, que é considerado como correspondente à capacidade de campo para solos com textura mais arenosa e com horizonte B textural (Reichardt, 1988) ou camada de impedimento ao fluxo de água. Contudo, observa-se que o potencial mátrico correspondente a essa densidade de fluxo variou de -5 a $-12,5 \mathrm{kPa}$, em função dos locais de amostragem.

A capacidade de campo estimada pelo método baseado na densidade de fluxo da água produz valores próximos aos do método baseado no potencial mátrico (Meyer \& Gee, 1999); entretanto, para os solos tropicais o potencial mátrico deve ser maior que $-33 \mathrm{kPa}$, como
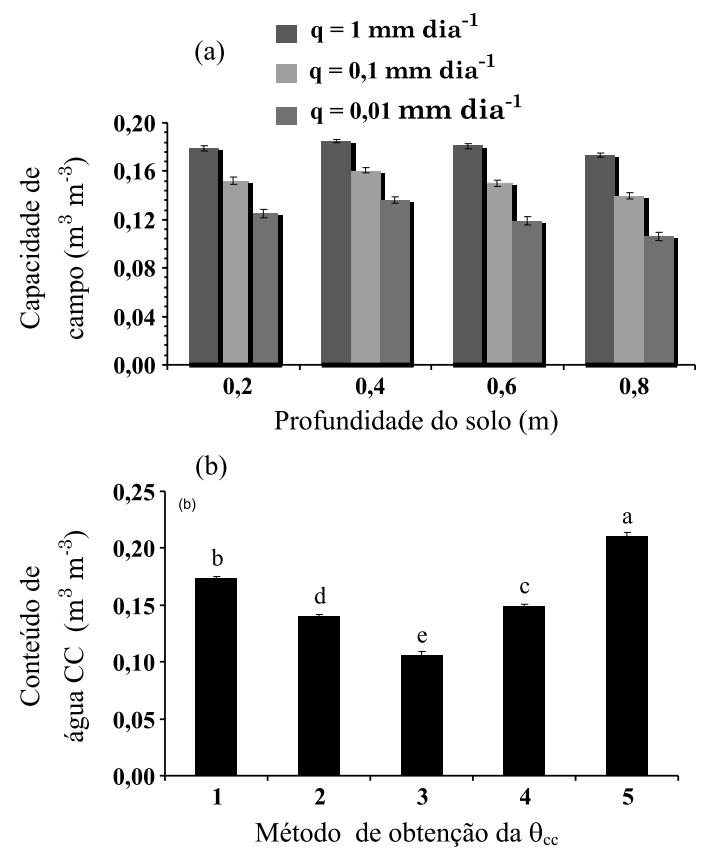

Figura 6. Capacidade de campo em função das densidades de fluxo $\left(1,0,0,1\right.$ e $\left.0,01 \mathrm{~mm} \mathrm{dia}^{-1}\right)$ nas profundidades de 0,$2 ; 0,4 ; 0,6$; e $0,8 \mathrm{~m}$ (a) e comparação da capacidade de campo, na profundidade de $0,8 \mathrm{~m}$, determinada pela: 1 ) densidade de fluxo de $1 \mathrm{~mm} \mathrm{dia}{ }^{-1}$; 2) densidade de fluxo de $0,1 \mathrm{~mm} \mathrm{dia}^{-1}$; 3) densidade de fluxo de $0,01 \mathrm{~mm} \mathrm{dia}^{-1}$; 4 e 5) potencial mátrico de $10 \mathrm{kPa}$, utilizando a curva de retenção elaborada com dados de campo e de laboratório, respectivamente (b). As médias foram comparadas pelo teste t a $5 \%$.

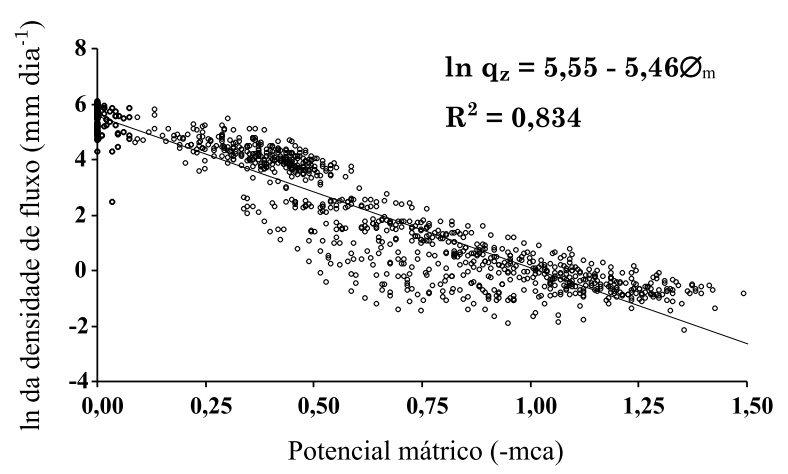

Figura 7. Densidade de fluxo da água $\left(\operatorname{Ln} q_{z}\right)$ determinada pela equação (2) (60 repetições) em função do potencial mátrico, para a profundidade de $0,80 \mathrm{~m}$.

sugere Reichardt (1988), uma vez que nesses solos há predomínio de argila de baixa atividade.

Os decréscimos da armazenagem correspondentes ao tempo necessário para alcançar densidade de fluxo da água de $1,0 \mathrm{~mm} \mathrm{dia}{ }^{-1}$ nas camadas de $0-0,2 ; 0$ 0,$4 ; 0-0,6$; e $0-0,8 \mathrm{~m}$, considerando a armazenagem de água máxima ajustada (Figura 8) para o tempo 
inicial (uma hora) de redistribuição, são de 20,8; 24 ; 26,4 ; e $28 \%$. No momento em que a densidade de fluxo da água na camada de $0-0,2 \mathrm{~m}$ atinge $1,0 \mathrm{~mm} \mathrm{dia}^{-1}$, nas demais camadas ela é de $1,94 \mathrm{~mm} \mathrm{dia}^{-1} ; 3,14 \mathrm{~mm} \mathrm{dia}^{-1}$; e 4,35 $\mathrm{mm} \mathrm{dia}^{-1}$. Observa-se que uma redução média de $25 \%$ da armazenagem máxima para esse solo reduz drasticamente a densidade de fluxo da água em todo o seu perfil, podendo-se assumir que o conteúdo de água correspondente a uma densidade de fluxo de 1,0 $\mathrm{mm} \mathrm{dia}^{-1}$ na camada superficial do solo pode ser considerado como a capacidade de campo, uma vez que nas camadas subsuperficiais elas são insignificantes, principalmente quando se considera que as condições de contorno aplicadas neste trabalho (impermeabilização das laterais da coluna de solo,

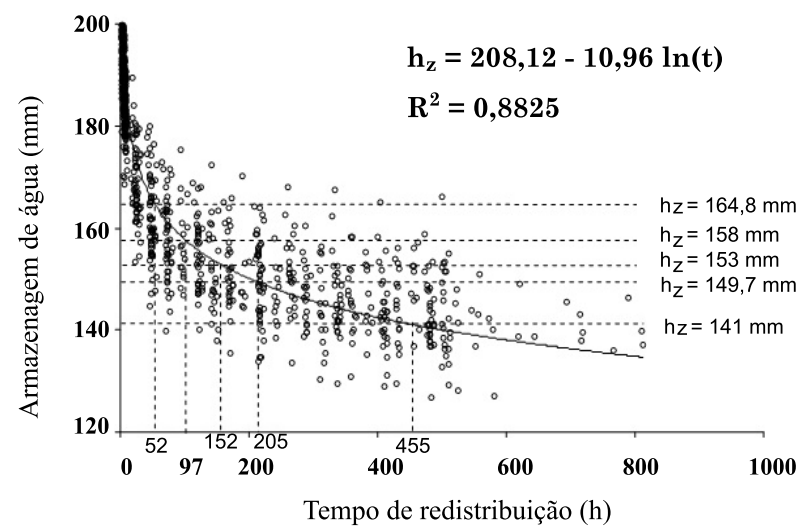

Figura 8. Armazenagem de água ao longo do tempo de redistribuição da água no solo para as $\mathbf{6 0}$ repetições e totalizando 965 pontos de medida. aplicação de grande volume de água e eliminação da entrada e saída de água pela superfície do solo) não são atingidas com muita frequência em condições naturais.

A distribuição da capacidade de campo relativa $\left(\mathrm{CC}_{\mathrm{r}}\right)$ ao conteúdo de água de saturação expressa a capacidade relativa de armazenagem de água no solo; o balanço ótimo entre a capacidade de água e a capacidade de ar na zona radicular do solo pode ser alcançado quando a $\mathrm{CC}_{\mathrm{r}}$ é maior ou igual a 0,6 e menor ou igual a 0,7 (Reynolds et al., 2008). Valores de $\mathrm{CC}_{\mathrm{r}}$ menores ou maiores que essa faixa resultam em atividade microbiana reduzida, devido aos insuficientes conteúdos de água ou ar no solo (Reynolds et al., 2008). Dessa forma, observa-se que, para as condições físicas da camada superficial $(0-0,4 \mathrm{~m})$, as $\mathrm{CC}_{\mathrm{r}}$ baseadas na capacidade de campo determinada para as densidades de fluxo de 0,1 e 1,0 $\mathrm{mm} \mathrm{dia}^{-1}$ estão compreendidas na faixa ótima (Figura 9).

No entanto, analisando todo o perfil do solo avaliado, é preferível optar pela $\mathrm{CC}_{\mathrm{r}}$ determinada com a densidade de fluxo de $1,0 \mathrm{~mm} \mathrm{dia}^{-1}$, visto que ela foi mais constante quanto à faixa recomendada.

\section{CONCLUSÕES}

1. O método recomendado para determinação da capacidade de campo do solo avaliado, Latossolo Vermelho-Amarelo textura média, é o baseado na densidade de fluxo da água de 1,0 $\mathrm{mm} \mathrm{dia}^{-1}$.

2. O potencial mátrico médio recomendado para determinação da capacidade de campo na profundidade de $0,8 \mathrm{~m}$, correspondente a uma densidade de fluxo de $1,0 \mathrm{~mm} \mathrm{dia}^{-1}$, é de $-10 \mathrm{kPa}$. (a)

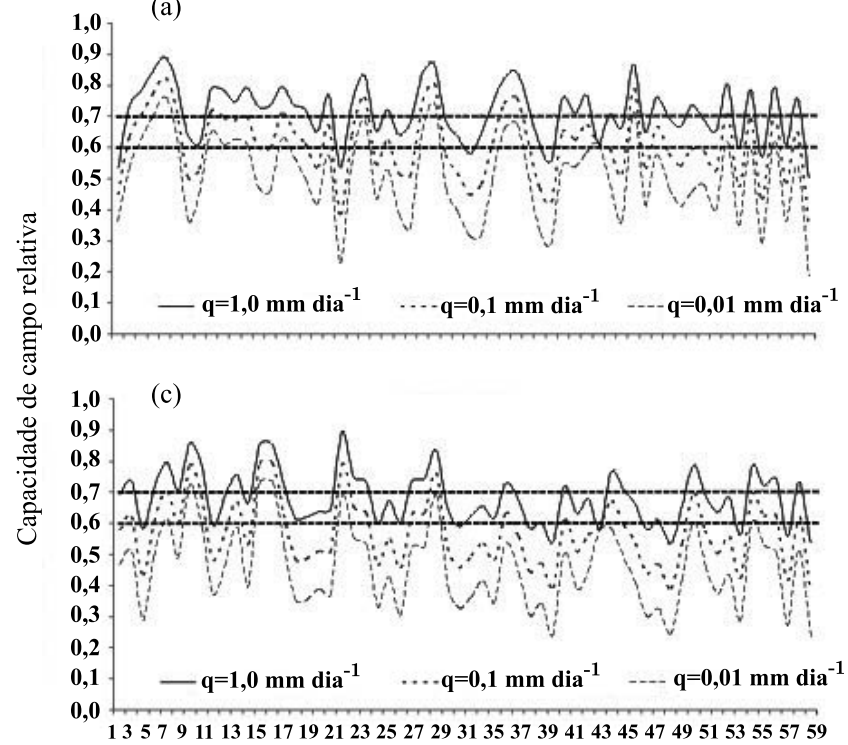

(b)

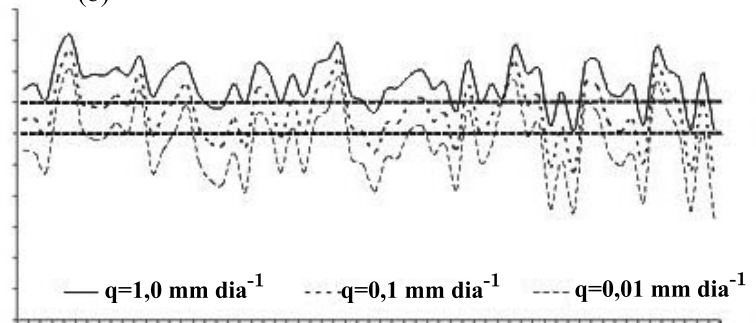

(d)

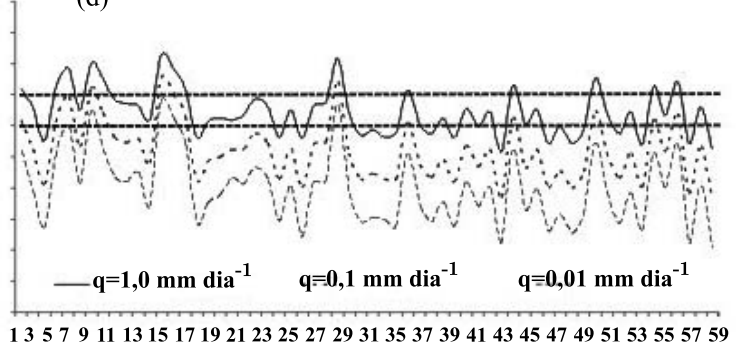

Local amostral

Figura 9. Variação espacial da capacidade de campo relativa nas profundidades de 0,2 (a), 0,4 (b), 0,6 (c) e $0,8 \mathrm{~m}(\mathrm{~d})$, correspondente às densidades de fluxo de $1,0,0,1 \mathrm{e} 0,01 \mathrm{~mm} \mathrm{dia}^{-1}$. 
3. A curva de retenção de água obtida no campo é mais recomendada que a obtida no laboratório para a estimativa do conteúdo de água no solo na capacidade de campo.

\section{AGRADECIMENTO}

Ao CNPq (Projeto Universal 477215/2007-6), pelo apoio financeiro.

\section{LITERATURA CITADA}

BRADY, N. \& WEIL, R.R. The nature and properties of soils. 13.ed. Prentice Hall, Upper Saddle River, 2002. 960p.

BRIGGS, L.J. \& McLANE, J.W. Moisture equivalent determinations and their application. Proc. Am. Soc. Agron., 2:138-147, 1910.

EMPRESA BRASILEIRA DE PESQUISA AGROPECUÁRIA . EMBRAPA. Centro Nacional de Pesquisa de Solos. Sistema brasileiro de classificação de solos. 2.ed. Brasília, Produção de Informação, 2006. 306p.

GEE, G.W. \& OR, D. Particle-size analysis. In: DANE, J.H. \& TOPP, G.C., ed. Methods of soil analysis: Physical methods. Madison, American Society of Agronomy, 2002. p.255289 .

GIVI, J.; PRASHER, S.O. \& PATEL, R.M. Evaluation of pedotransfer functions in predicting the soil water contents at field capacity and wilting point. Agric. Water Manag., 70:83-96, 2004.

JABRO, J.D.; EVANS, R.G.; KIM, Y. \& IVERSEN, W.M. Estimating in situ soil-water retention and field water capacity in two contrasting soil textures. Irrig. Sci., 27:223$229,2009$.

KOOREVAAR, P.; MENELIK, G. \& DIRKSEN, C. Elements of soil physics. Amsterdan: Elsevier, 1999. 228p.
MELO FILHO, J.F. \& LIBARDI, P.L. Estabilidade temporal de medidas do teor e do potencial mátrico da água no solo em uma transeção. R. Bras. Ci. Solo, 29:497-506, 2005.

MEYER, P.D. \& GEE, G.W. Flux-based estimation of field capacity. J. Geotechn. Geoenviron. Eng., 125:595-599, 1999.

MORGAN, K.T.; PARSONS, L.R. \& WHEATON, T.A. Comparison of laboratory- and field-derived soil water retention curves for a fine sand soil using tensiometric, resistance and capacitance methods. Plant Soil, 234:153157, 2001.

NACHABE, M.H. Refining the definition of field capacity in the literature. J. Irrig. Drainage Eng-ASCE, 124:230-232, 1998.

REICHARDT, K. Capacidade de campo. R. Bras. Ci. Solo, 12:221-216, 1988.

REYNOLDS, W.D.; DRURY, C.F.; YANG, X.M. \& TAN, C.S. Optimal soil physical quality inferred through structural regression and parameter interactions. Geoderma, 146:466-474, 2008.

RICHARDS, L.A. \& WEAVER, L.R. Moisture retention by some irrigated soils as related to soil moisture tension. J. Agric. Res., 69:215-235, 1944.

RUIZ, H.A.; FERREIRA, G.B. \& PEREIRA, J.B.M. Estimativa da capacidade de campo de Latossolos e Neossolos quartzarênicos pela determinação do equivalente de umidade. R. Bras. Ci. Solo, 27:389-393, 2003.

TWARAKAVI, N.K.C.; SAKAI, M. \& SIMUNEK, J. An objective analysis of the dynamic nature of field capacity. Water Res. Res., 45:1-9, 2009.

van GENUCHTEN, M.T. A Closed-form equation for predicting the hydraulic conductivity of unsaturated soils. Soil. Sci. Soc. Am. J., 44:892-898, 1980.

VEIHMEYER, F.J. \& HENDRICKSON, A.H. The moisture equivalent as a measure of the field capacity of soils. Soil Sci., 32:181-193, 1931. 